\title{
Orbital Lymphatic-Venous Malformation Accompanied by an Intraocular Vascular Malformation: A Rare Case Study
}

\author{
Karny Shouchane-Blum ${ }^{a, b} \quad$ Iftach Yassur $^{a, b} \quad$ Amir Sternfeld $^{b, c}$ \\ Miriam Regev ${ }^{b, d}$ Orly Gal-Or ${ }^{a, b} \quad$ Liora Kornreich $^{\text {b, e }}$ Rita Ehrlich ${ }^{a}$ b \\ Miriam Ehrenberg ${ }^{b} c$ \\ aDepartment of Ophthalmology, Rabin Medical Center - Beilinson Hospital, Petach Tikva, \\ Israel; bSackler Faculty of Medicine, Tel Aviv University, Tel Aviv, Israel; ' Department of \\ Ophthalmology, Schneider Children's Medical Center of Israel, Petah Tikva, Israel; ${ }^{\mathrm{d}}$ The \\ Danek Gertner Institute of Human Genetics, Sheba Medical Center, Tel-Hashomer, Israel; \\ eImaging Department, Schneider Children's Medical Center of Israel, Petah Tikva, Israel
}

\section{Keywords}

LMV - Lymphatic-venous-malformation · Lymphangioma $\cdot$ RNF213 gene - Intraocular vascular anomaly

\section{Abstract}

Lymphatic-venous malformations (LVMs) are development defects that result in abnormal connections between the lymphatic and venous systems. The authors describe a 7-weeks-old female infant who presented with a right orbital LVM extending to the ipsilateral cheek and subconjunctiva of the right eye, intracranial developmental venous anomalies in the right cerebellum, and a significant right eye intraocular retinal vascular malformation. Since orbital LVM is usually diagnosed in infancy or childhood, pediatric ophthalmologists should actively look for intraocular vascular malformations as such findings can poorly affect a patient's vision.

\section{Introduction}

Lymphatic-venous malformations (LVMs) [1], also known as lymphangiomas, are a subgroup of slow-flow vascular malformations [2]. They are developmental defects caused by an improper connection between the lymphatic and venous system or abnormal development 
of lymphatic vessels. LVMs are considered benign tumors, usually present at birth, that continue to grow at the normal rate of endothelial turnover throughout the subject's life [3]. The most common ( $\sim 60 \%$ ) locations of LVMs are in the head and neck, but they can be found in any body part where lymphatic vessels are present [4-6].

Orbital LVMs account for about 1-3 percent of orbital tumors [7]. They typically present in childhood in the subconjunctival and periocular tissues. However, orbital LVMs can extend to the frontotemporal region and the cheek and may be associated with intracranial vascular malformations, such as venous anomalies [8-10]. Lesions can be asymptomatic until they reach a large size, or intralesional hemorrhage occurs and causes swelling, proptosis, decreased motility of the eye, or pain [11]. Even though they are benign, these lesions can threaten vision by causing a compressive optic neuropathy [7]. We present a rare case in which an orbital LVM is accompanied by a significant retinal vascular disorder in the ipsilateral eye.

\section{Case Report}

A 7-weeks-old female was referred to our clinic because of an abnormal red reflex of the right eye. General development was appropriate to age. Pregnancy with the patient was achieved via IVF and sperm donation. The subject's mother, Jewish of Iraqi-Ashkenazi origin, and sperm donor of Danish origin have no known ocular disorders (self-reported). Delivery was induced at 39 weeks of gestation due to preeclampsia and was traumatic (vacuum-assisted delivery, fractured clavicle, and meconium-stained amniotic fluid). The birth weight was 3,490 g.

On her exam, the subject blinked to light and had normal anterior segments in each eye. After dilation, a dense cloudy vitreous was seen in the right eye that obscured the posterior pole and did not enable visualization of the optic nerve or any other details of the posterior pole (shown in Fig. 1). The left eye had normal optic disc appearance, normal foveal reflex, and attached retina.

Examination under anesthesia (EUA) revealed a normal anterior segment in both eyes. After pupil dilation, RetCam images were taken, and an ultrasound showed an elevated, dome-shaped lesion (mass) in the macular area compatible with organized vitreous hemorrhage. Left fundus appeared normal.

Due to swelling of the right cheek (shown in Fig. 2), the patient underwent MRI, magnetic resonance angiography, and magnetic resonance venography of the brain and orbits. Imaging revealed a LVM in the right orbit extending anteriorly to the soft tissues of the right cheek and

Fig. 1. Right eye fundus photo demonstrating central opacity blocking visibility of the posterior pole.

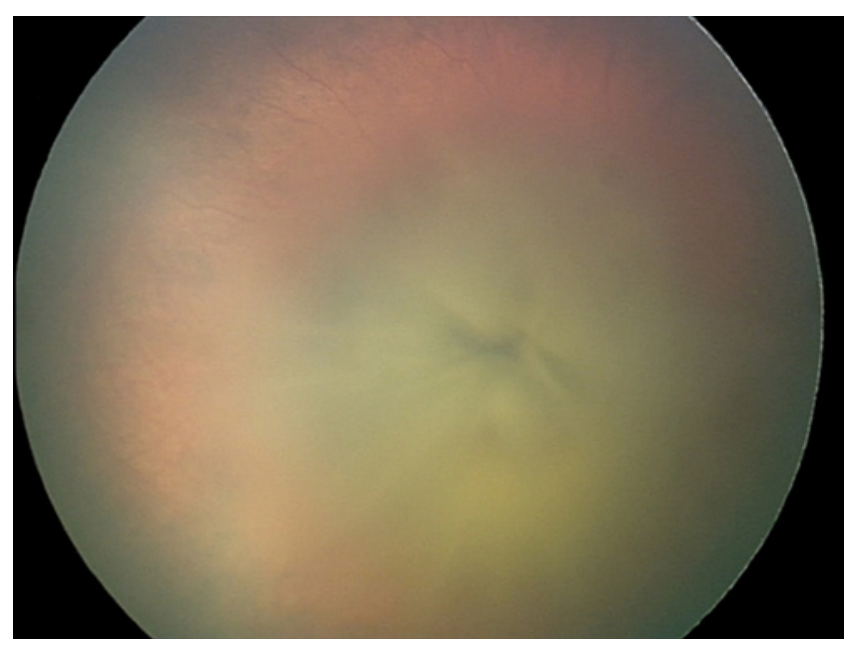


Shouchane-Blum et al.: Orbital Lymphatic-Venous-Malformation and Intraocular Vascular Malformation

Fig. 2. Photo of the facial features: notice asymmetry with right cheek fullness (arrow), secondary to LVM. LVM, lymphaticvenous malformation.
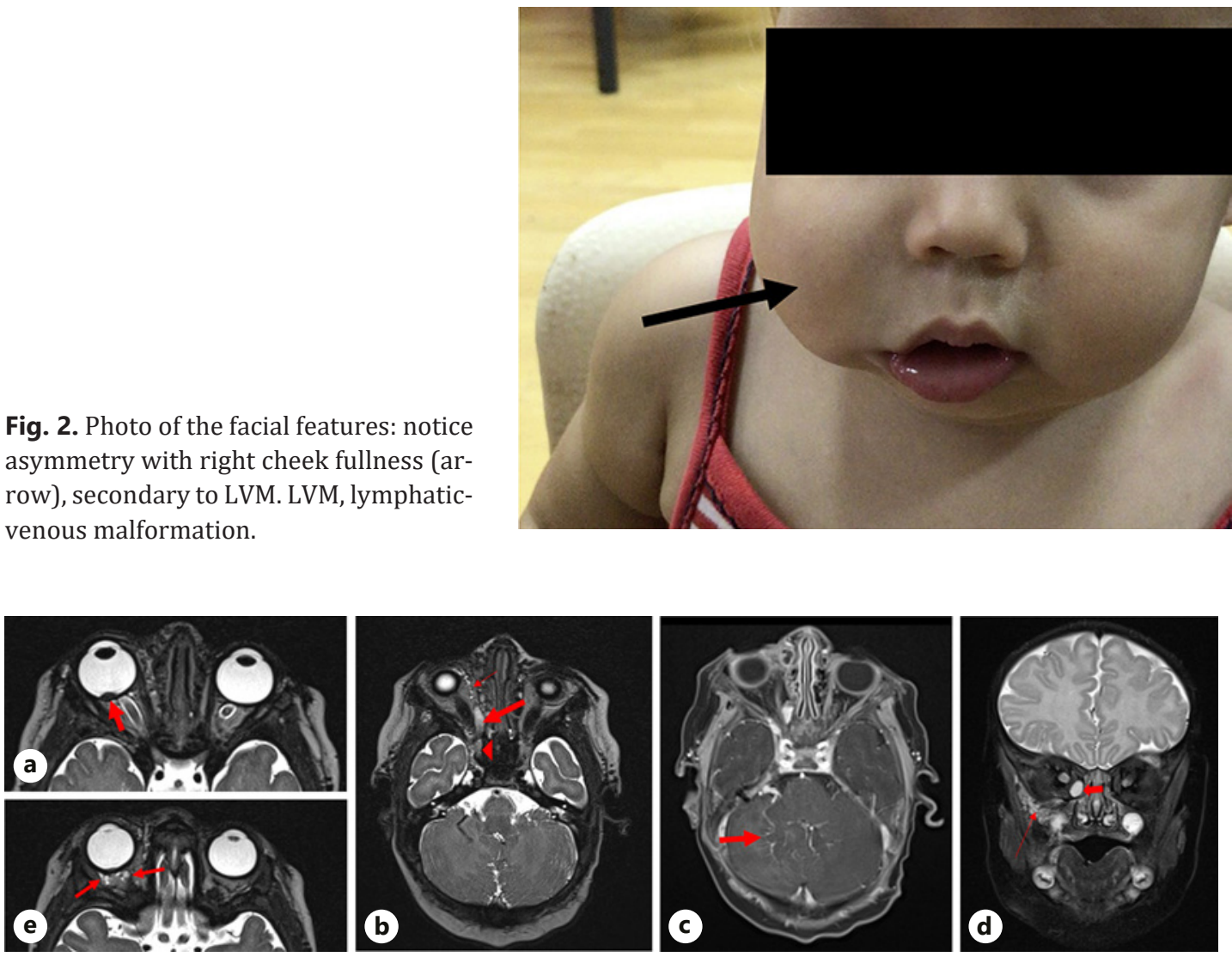

Fig. 3. a Axial T2 weighted image of the orbits. Right eye: a small hypointense mass, compatible with hemorrhage, is seen protruding into the vitreous (arrow). b Axial T2 weighted image. Right orbit - several components of the LVM are seen: a hyperintense tubular mass in the apex of the right orbit (large arrow) extending into the cavernous sinus (arrowhead), small hyperintense lesions extending from the eyelid into the medial extraconal space (small arrow). c Axial T1 weighted image post contrast injection. A venous anomaly is seen in the right cerebellum (arrow). d Coronal T2 weighted image. Extension of the LVM into the right periorbital region is seen (arrow). Note also the medial extraconal component (thick arrow). e Axial T2 weighted image of the orbits. On the right, small hyperintense lesions compatible with retrobulbar extension of the LVM are seen (arrows). LVM, lymphatic-venous malformation.

posteriorly to the right cavernous sinus. Infiltration of the LVM behind the eyeball caused a slight flattening of the posterior aspect of the eyeball, along with slight forward displacement of the eyeball. In the brain, there was a venous anomaly in the right cerebellum (shown in Fig. 3).

The patient underwent a vitrectomy in her right eye to clear the old dense vitreal hemorrhage without any further interactions during surgery. Subsequent fluorescein angiography demonstrated a subretinal lesion in the posterior pole accompanied by capillary nonperfusion and microaneurysms at the retinal periphery $360^{\circ}$ (shown in Fig. 4). Therefore, laser coagulation to the retinal periphery was performed.

A follow-up EUA also revealed a transparent nasal subconjunctival mass compatible with LVM (shown in Fig. 5), a central macular scar, and peripheral retinal laser scars in the right eye. The patient also underwent genetic evaluation. Whole-exome sequencing tests of the subject and her mother revealed a maternal inherited NM_001256071.1:c.13798-2A>C variant in the RNF213 gene (see online suppl. material 1; for all online suppl. material, see 
Shouchane-Blum et al.: Orbital Lymphatic-Venous-Malformation and Intraocular Vascular Malformation

www.karger.com/doi/10.1159/000515272). The mother had a normal physical exam but did not complete the recommended clinical workup, including a dilated eye exam. However, she has good visual acuity in both eyes.

The patient is now almost 2 years old and still continues regular follow-up visits in our clinic. She is continuously treated for amblyopia and seems to develop useful visual acuity in the right eye. She did not undergo any other surgical procedure to date.

\section{Discussion}

Presented is a unique case of co-occurrence of facial, orbital, and subconjunctival LVM, associated with a venous anomaly, and accompanied by an intraocular retinal vascular anomaly and vitreous hemorrhage in the ipsilateral eye of a baby girl with a genetic molecular change in her $R N F 213$ gene. In the past, it was assumed there were no lymphatic vessels in the eye [12]. However, there have been significant advances in lymphatic research in recent years. Studies now support the presence of lymphatic components in the central nervous system, orbit, and inside the eye [13-15]. Yücel et al. [13] demonstrated the presence of lymphatic vessels in the ciliary body and confirmed drainage of radioactively labeled albumin from the anterior chamber to cervical, retropharyngeal, submandibular, and preauricular lymph nodes. Dickinson and Gausas [14] demonstrated the presence of lymphatic vessels in the dura surrounding the optic nerve.

Katz et al. [8] previously reported the association of orbital LVMs with noncontiguous intracranial venous anomalies in 28 percent of patients primarily diagnosed with orbital LVM. This association indicates a propensity for multifocal anomalous vascular formation. In their paper, the authors perceive LVM of the orbit as a spectrum of multicentric venous LVMs, probably depending on time of occurrence during embryonal development.

Two cases previously published [16] described orbital LVM accompanied by iris venous malformation, abnormal branching of retinal vessels, abnormal foveal reflex, and poor vision. The currently presented patient had extensive retinal vascular involvement that included the macula and the periphery, probably representing an extreme scenario of the continuum suggested. We suggest that the same embryonal sequence that induced the abnormal vascular development on the same side of the brain, orbit, and face also influenced the intraocular vasculature anomalous development, which caused very early vitreal hemorrhage and capillary nonperfusion.

Finally, our patient had a genetic variant in the RNF213 gene. Mutations in RNF213 are known to be associated with moyamoya disease, an idiopathic vascular disorder of intracranial
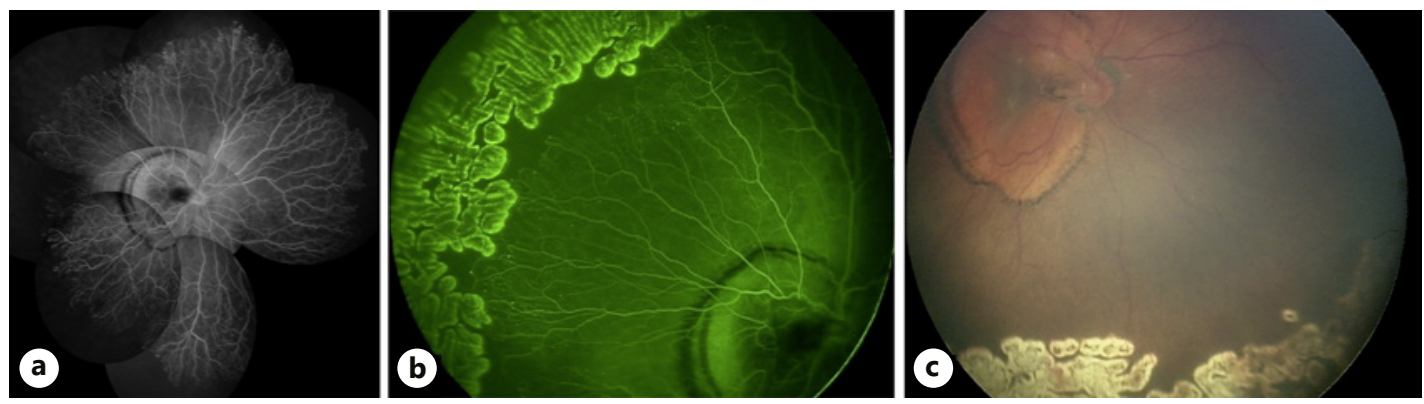

Fig. 4. FA of the right eye demonstrating subretinal lesion in the posterior pole after vitrectomy and retinal periphery CNP (a); FA of the right eye post vitrectomy demonstrating macular scar and peripheral laser therapy scars (b); and fundus photo of the right eye post vitrectomy demonstrating macular scar and peripheral laser therapy scars (c). FA, fluorescein angiography; CNP, capillary nonperfusion. 
Shouchane-Blum et al:: Orbital Lymphatic-Venous-Malformation and Intraocular Vascular Malformation

Fig. 5. Right eye nasal subconjunctival LVM (arrow). LVM, lymphatic-venous malformation.

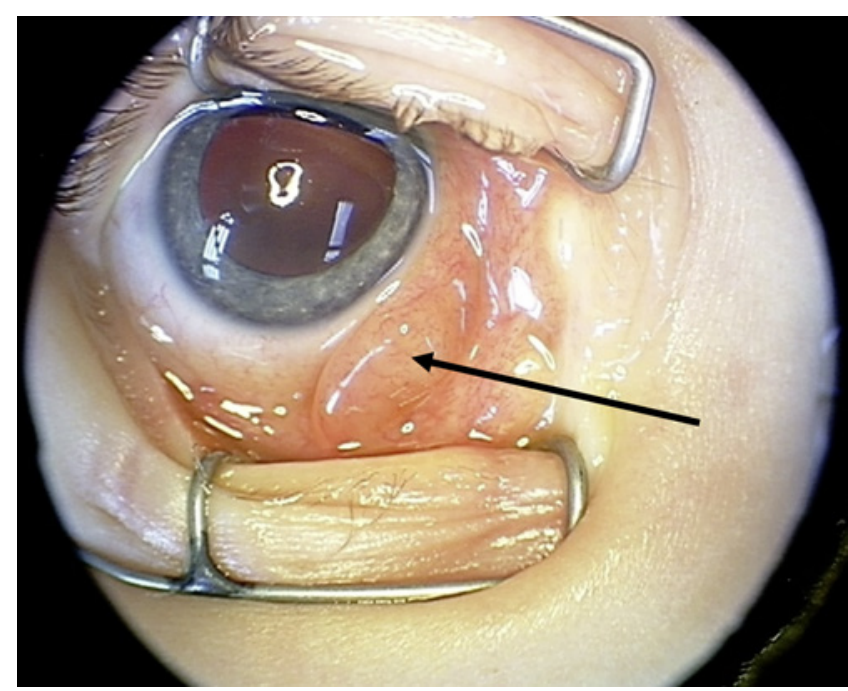

arteries. The inheritance may be autosomal recessive or autosomal dominant with incomplete penetrance. The c.13798-2A>C variant occurs in a highly conserved splice site area that has not been described previously in the literature. This variant has a very low frequency in healthy populations, 1: 0.0000041, as estimated by gnomAD. Altogether, the finding was concluded as a variant of unknown significance. Relevant clinical workup was recommended to the mother but has not yet been completed. Interestingly, RNF213 knockdown zebra fish showed severely abnormal sprouting vessels in the head region $[17,18]$. However, no direct association has yet been found between this gene and LVMs in humans.

In conclusion, we suggest that in cases of diffuse orbital LVM, retinal vascular abnormalities should be prospectively sought. Since LVMs are most frequently diagnosed in childhood, EUAs, including fluorescein angiographies, should be undertaken. Further clinical investigation is needed to understand the frequency and severity of intraocular anomalous vascular component as part of diffuse orbital LVMs associated with venous anomalies, and the significance of the variant in the RNF213 gene.

\section{Acknowledgements}

We would like to thank Dr. Tamar Steinberg for closely following this case and integrating all medical treatments.

\section{Statement of Ethics}

Written informed consent was obtained from the patient's parent, who is her legal guardian, for publication of this case report and any accompanying images.

\section{Conflict of Interest Statement}

The authors declare that they have no competing interests. 
Shouchane-Blum et al.: Orbital Lymphatic-Venous-Malformation and Intraocular Vascular Malformation

\section{Funding Sources}

This research did not receive any specific grant from funding agencies in the public, commercial, or not-for-profit sectors.

\section{Author Contributions}

Ophthalmological material preparation and data collection were performed by K.S.B., I.Y., O.G., R.E., A.S., and M.E. Radiological material preparation and data collection were performed by L.K. Genetical material preparation and data collection were performed by M.R. The first draft of the manuscript was written by K.S.B. and M.E., and all the authors commented on previous versions of the manuscript. All the authors read and approved the final manuscript.

\section{References}

1 International Society for the Study of Vascular Anomalies. ISSVA classification for vascular anomalies Available from: https://www.issva.org/UserFiles/file/ISSVA-Classification-2018.pdf. Published 2014 Apr. Updated 2018 May. Accessed 2020 0ct 21.

2 Mulliken JB, Glowacki J. Hemangiomas and vascular malformations in infants and children: a classification based on endothelial characteristics. Plast Reconstr Surg. 1982 Mar;69(3):412-22.

3 Ethunandan M, Mellor TK. Haemangiomas and vascular malformations of the maxillofacial region: a review. Br J Oral Maxillofac Surg. 2006 Aug;44(4):263-72.

4 Wiegand S, Eivazi B, Barth PJ, von Rautenfeld DB, Folz BJ, Mandic R, et al. Pathogenesis of lymphangiomas. Virchows Arch. 2008 Jul;453(1):1-8.

5 Waner M, O TM. Multidisciplinary approach to the management of lymphatic malformations of the head and neck. Otolaryngol Clin North Am. 2018 Feb;51(1):159-72.

6 Wiegand S, Ott A, Zimmermann AP, Wilhelm T, Eivazi B, Werner JA, et al. Localization of lymphatic malformations of the neck. Lymphat Res Biol. 2013 Jun;11(2):101-3.

7 Nassiri N, Rootman J, Rootman DB, Goldberg RA. Orbital lymphaticovenous malformations: current and future treatments. Surv Ophthalmol. 2015 Sep-Oct;60(5):383-405.

8 Katz SE, Rootman J, Vangveeravong S, Graeb D. Combined venous lymphatic malformations of the orbit (so-called lymphangiomas). Association with noncontiguous intracranial vascular anomalies. Ophthalmology. 1998 Jan;105(1):176-84.

9 Bisdorff A, Mulliken JB, Carrico J, Robertson RL, Burrows PE. Intracranial vascular anomalies in patients with periorbital lymphatic and lymphaticovenous malformations. AJNR Am J Neuroradiol. 2007 Feb;28(2):335-41.

10 Gürelik M, Ozüm U, Erdoğan H, Aslan A. Orbital lymphangioma and its association with intracranial venous angioma. Br J Neurosurg. 2004 Apr;18(2):168-70.

11 Woo YJ, Kim CY, Sgrignoli B, Yoon JS. Orbital lymphangioma: characteristics and treatment outcomes of 12 cases. Korean J Ophthalmol. 2017 Jun;31(3):194-201.

12 Bill A. Blood circulation and fluid dynamics in the eye. Physiol Rev. 1975 Jul;55(3):383-417.

13 Yücel YH, Johnston MG, Ly T, Patel M, Drake B, Gümüş E, et al. Identification of lymphatics in the ciliary body of the human eye: a novel "uveolymphatic" outflow pathway. Exp Eye Res. 2009 Nov;89(5):810-9.

14 Dickinson AJ, Gausas RE. Orbital lymphatics: do they exist? Eye. 2006 Oct;20(10):1145-8.

15 Mathieu E, Gupta N, Ahari A, Zhou X, Hanna J, Yücel YH. Evidence for cerebrospinal fluid entry into the optic nerve via a glymphatic pathway. Invest Ophthalmol Vis Sci. 2017 Sep;58(11):4784-91.

16 Vavvas D, Fay A, Watkins L. Two cases of orbital lymphangioma associated with vascular abnormalities of the retina and iris. Ophthalmology. 2004 Jan;111(1):189-92.

17 Liu W, Morito D, Takashima S, Mineharu Y, Kobayashi H, Hitomi T, et al. Identification of RNF213 as a susceptibility gene for moyamoya disease and its possible role in vascular development. PLoS One. 2011 Jul;6(7): e22542.

18 Miyatake S, Miyake N, Touho H, Nishimura-Tadaki A, Kondo Y, Miyatake S, et al. Homozygous c.14576G>A variant of RNF213 predicts early-onset and severe form of moyamoya disease. Neurology.2012 Mar;78:803-10. 